\title{
Bailout embeddings, targeting of invariant tori, and the control of Hamiltonian chaos
}

\author{
Julyan H. E. Cartwright, ${ }^{1, *}$ Marcelo O. Magnasco, ${ }^{2, \dagger}$ and Oreste Piro ${ }^{3, *}$ \\ ${ }^{1}$ Laboratorio de Estudios Cristalográficos, CSIC, E-18071 Granada, Spain \\ ${ }^{2}$ Mathematical Physics Laboratory, Rockefeller University, Box 212, 1230 York Avenue, New York, New York 10021 \\ ${ }^{3}$ Institut Mediterrani d'Estudis Avançats, CSIC-UIB, E-07071 Palma de Mallorca, Spain
}

(Received 22 September 2001; revised manuscript received 20 December 2001; published 3 April 2002; publisher error corrected 9 April 2002)

\begin{abstract}
We introduce a technique, which we term bailout embedding, that can be used to target orbits having particular properties out of all orbits in a flow or map. We explicitly construct a bailout embedding for Hamiltonian systems so as to target invariant tori. We show how the bailout dynamics are able to lock onto extremely small regular islands in a chaotic sea.
\end{abstract}

\section{DOI: 10.1103/PhysRevE.65.045203}

Control of chaos in nonlinear dynamical systems has been achieved by applying small perturbations that effectively change the dynamics of the system around the regiontypically a periodic orbit—that one wishes to stabilize [1]. This method has been successful in dissipative systems, but its extensions to control and targeting in Hamiltonian systems [2-11] have met various difficulties not present in the dissipative case: the absence of attracting sets, for instance, makes it hard to stabilize anything. In addition, these methods require that one know beforehand what one wants to do; in particular, the orbit to be stabilized may have to be known rather accurately in advance.

In this Rapid Communication we present a technique allowing us to control Hamiltonian chaos, in such a way as to keep the original dynamics intact, but that shifts the stability of different kinds of orbits in the dynamics. We do so by embedding our Hamiltonian system within a larger space, meaning we augment the number of degrees of freedom, keeping an intact copy of the original system on one privileged slice; all of the control is achieved through use of the perpendicular directions to this intact copy. We call this method a bailout embedding for reasons that will promptly become clear. We apply the technique to an extremely hard and hitherto close to intractable problem in chaos control of Hamiltonian systems: selecting small Kol'mogorov-Arnol'dMoser (KAM) islands within chaotic seas in systems that are almost ergodic. We show below how our technique is able to find and render asymptotically stable minute islands of order within a map. While formerly this could be done by sophisticated algorithms on the basis of complex logic, our method distills the complexity of this calculation into a simple, forward-iterated dynamical system. Thus our method allows us to stabilize KAM islands without knowing their locations in advance.

A classical problem in Hamiltonian dynamics is locating invariant or KAM tori. Hamiltonian systems exist between two opposite extremes, of fully integrable systems and fully

\footnotetext{
*URL: http://lec.ugr.es/ julyan. Electronic address: julyan@lec.ugr.es

${ }^{\dagger}$ URL: http://asterion.rockefeller.edu/. Electronic address: marcelo@sur.rockefeller.edu

ॠURL: http://www.imedea.uib.es/ piro. Electronic address: piro@imedea.uib.es
}

PACS number(s): $05.45 . \mathrm{Gg}$

ergodic ones. Fully integrable systems are characterized by dynamics unfolding on invariant tori. The KAM theorem asserts that, as a parameter taking the system away from integrability is increased, these tori break and give rise to chaotic regions in a precise sequence; for any particular value of this parameter in a neighborhood of the integrable case, there are surviving tori. The problem with finding them is that, the dynamics being volume preserving, merely evolving trajectories either forward or backward does not give us convergence onto tori [12], and since, for large values of the nonlinearity, they cover a very small measure of the phase space, locating them becomes an extremely difficult problem. Moreover, there is the further problem that even if we start on an island, we should be able to recognize it as such. In fact, several sophisticated analytical and semianalytical techniques have had to be developed to assist in the search and characterization of KAM tori in highly nonlinear Hamiltonian systems (see, for instance, [13]). A bailout embedding solves these problems by transforming the KAM trajectories into global attractors of the embedded system; finding them is now independent of the choice of initial conditions.

Our technique is based upon the dynamics of a small neutrally buoyant sphere-a passive scalar-in an incompressible fluid flow $\boldsymbol{u}$ [14]. Under assumptions allowing us to retain only the Bernoulli, Stokes drag, and Taylor added mass contributions to the force exerted by the fluid on the sphere, the equation of motion for the sphere at the position $x$ is

$$
\frac{d}{d t}[\dot{\boldsymbol{x}}-\boldsymbol{u}(\boldsymbol{x})]=-(\lambda I+\nabla \boldsymbol{u}) \cdot[\dot{\boldsymbol{x}}-\boldsymbol{u}(\boldsymbol{x})]
$$

so that the difference between the particle velocity and the velocity of the surrounding fluid is exponentially damped with damping coming from $-(\lambda+\boldsymbol{\nabla} \boldsymbol{u})$. However, in the case in which the flow gradients reach the magnitude of the viscous drag coefficient, there is the possibility that around hyperbolic stagnation points the Jacobian matrix $\boldsymbol{\nabla} \boldsymbol{u}$ may acquire a positive eigenvalue in excess of the drag coefficient. In these instances, the trajectories of these passive scalars, instead of converging exponentially onto $\dot{\boldsymbol{x}}=\boldsymbol{u}$, detach from such trajectories. The result is that the passive scalars explore practically all of the flow, but tend to avoid regions of high shear.

Equation (1) is but one instance of a more general structure. Let us consider a flow of the form $\dot{x}=f(x)$. If we take 


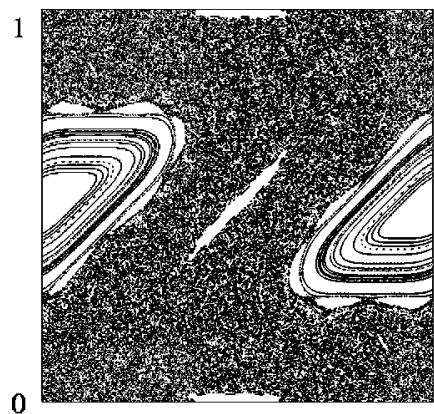

0

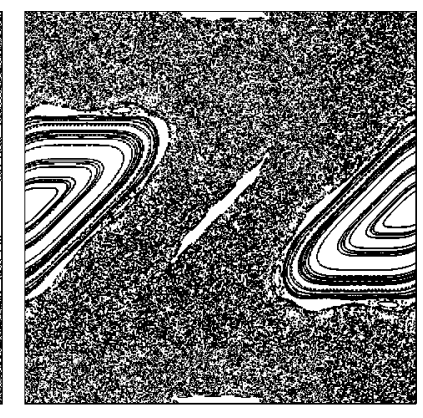

10

(a)

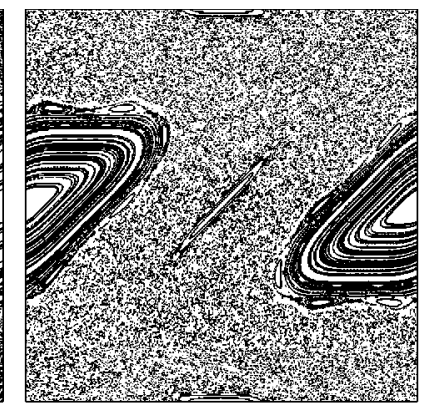

10

(b)

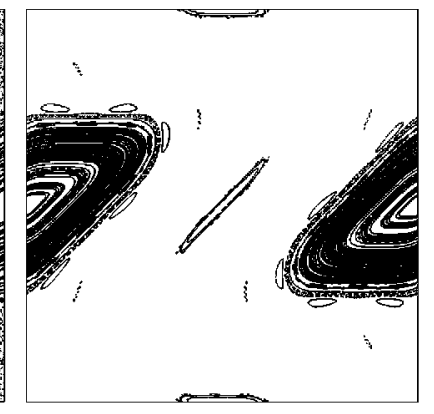

10

(c)

(d)

FIG. 1. The bailout embedding can target KAM orbits in the standard map at $k=2.100$ random initial conditions were chosen and iterated for 10000 steps; the next 1000 iterations are shown on the unit torus. (a) Original map, (b) $\lambda=1$, (c) $\lambda=0.6$, (d) $\lambda=0.5$.

its time derivative, we obtain a different flow, $\ddot{x}=f^{\prime}(x) \dot{x}$. It is different because, being second order, it exists in a larger space and has many solutions that are not solutions of the smaller one. Still, the original equation is contained within the larger system, in the sense that every solution of $\dot{x}$ $=f(x)$ is a solution of $\ddot{x}=f^{\prime}(x) \dot{x}$. We may say that $\dot{x}$ $=f(x)$ is embedded within $\ddot{x}=f^{\prime}(x) \dot{x}$. There are infinitely many ways to embed; for example, $\ddot{x}=f^{\prime}(x) f(x)$ is also an embedding, but it is clearly inequivalent to the first.

Of course, embedding a system changes notions of stability, because stability refers to perturbations, and in a larger system there are all of the old perturbations plus a batch of new ones. So, even though all the solutions of the original system are preserved, by adding new directions away from the old solutions we may transform formerly stable solutions into unstable ones in the larger setting; see, for example, studies of the manifold bubbling transition [15]. The trivial way to embed a system is through a cross product; for instance, $\dot{x}=f(x), x \in \mathbb{M}$, is embedded within $\mathbb{M} \times \mathbb{R}$ as

$$
\begin{gathered}
\dot{x}=f(x)+g(x, y), \\
\dot{y}=\alpha y,
\end{gathered}
$$

where $g(x, y)$ is arbitrary except for requiring that $g(x, 0)$ $\equiv 0$, which guarantees that for $y=0$ we have the original system. If $\alpha<0$ then $y$ always dies out, so we necessarily recover the original object; in this case, we can call the embedding itself stable, in the sense that any motion away from the embedded object takes us back to it. Both of the derivative embeddings in the previous paragraph were unstable; stable versions can be constructed rather simply, for instance,

$$
\frac{d}{d t}[\dot{x}-f(x)]=-\kappa[\dot{x}-f(x)]
$$

of which the previous examples were the $\kappa=0$ limit. This embedding ensures that for any initial condition the distance between the actual trajectory and the embedding diminishes exponentially with time. Equation (3) begins to resemble Eq. (1).

We define a bailout embedding as one of the form

$$
\frac{d}{d t}[\dot{x}-f(x)]=-\kappa(x)[\dot{x}-f(x)],
$$

where $\kappa(x)<0$ on a set of orbits that are unwanted, and $\kappa(x)>0$ otherwise. Thus the natural behavior of a bailout embedding is that the trajectories in the full system tend to detach or bail out from the embedded subsystem into the larger space, where they bounce around. If these orbits reach a stable region of the embedding, $\kappa(x)>0$, they once again collapse onto the original dynamical system. In this way we can create a larger version of the dynamics in which specific sets of orbits are removed from the asymptotic set, while preserving the dynamics of another set of orbits-the wanted or targeted one-as attractors of the enlarged dynamical system. For the special choice of $\kappa(x)=-\left(\lambda+\partial_{x} f\right)$, where $\partial_{x}$ denotes the derivative at the point $x$, analogous to the fluid dynamics of a passive scalar described by Eq. (1), these dynamics were shown in [14] to detach from saddle points and other unstable regions in conservative dynamics.

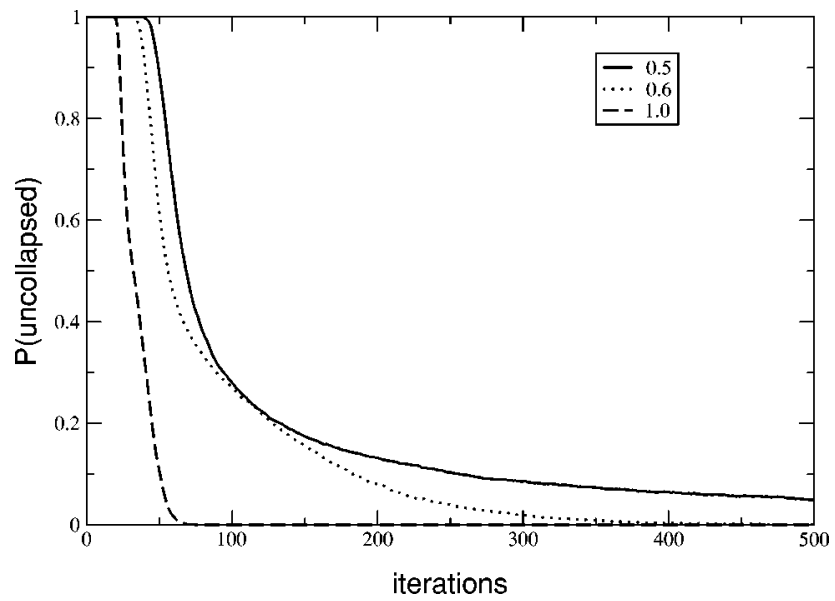

FIG. 2. Random initial conditions eventually collapse onto the original dynamics. Here we plot the fraction of initial conditions such that $\left|x_{n+1}-f\left(x_{n}\right)\right|>10^{-10}$ as a function of the number of iterations elapsed, for 8000 random initial conditions in the standard map at $k=2$. The three curves correspond to cases (b), (c), and (d) in Fig. 1. 


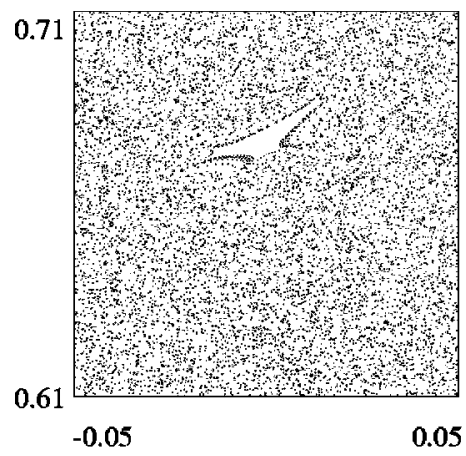

(a)

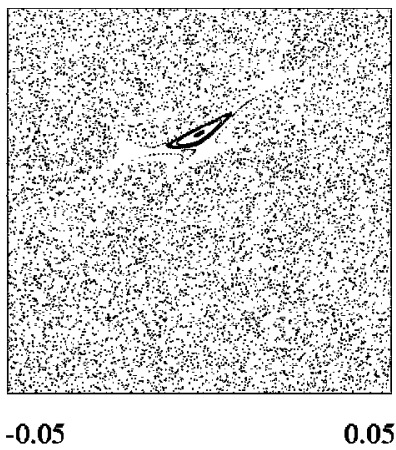

(b)

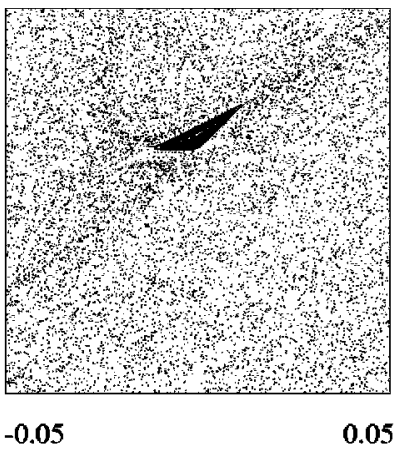

(c)

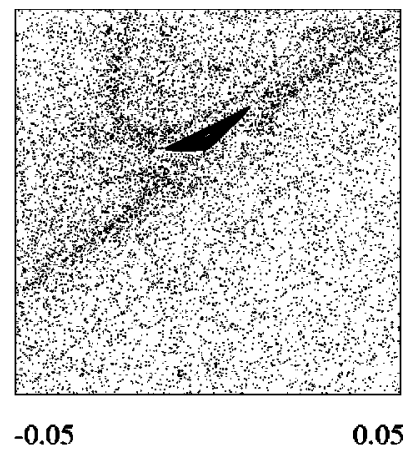

(d)

FIG. 3. The bailout process can find extremely small KAM islands. The standard map for $k=7$ has a chaotic sea covering almost the entire torus, except for a tiny period-2 KAM torus near position $(0,0.6774) .1000$ random initial conditions were chosen and iterated for 20000 steps; the next 1000 iterations are shown. (a) Original map, (b) $\lambda=1.4$, (c) $\lambda=1.3$, (d) $\lambda=1.2$.

It is not hard to extend flow bailout, Eq. (4), to maps in the obvious fashion. Given a map $x_{n+1}=T\left(x_{n}\right)$ the bailout embedding is given by

$$
x_{n+2}-T\left(x_{n+1}\right)=K\left(x_{n}\right)\left[x_{n+1}-T\left(x_{n}\right)\right],
$$

provided that $|K(x)|>1$ over the unwanted set. (In the map system, almost any expression written for the flow translates to something close to an exponential; in particular, stability eigenvalues have to be negative in the flow case to represent stability, while they have to be smaller than 1 in absolute value in the map case.) The particular choice of the gradient as the bailout function $\kappa(x)=-\left(\lambda+\partial_{x} f\right)$ in a flow translates in the map setting to $K(x)=e^{-\lambda} \partial_{x} T$.

A classical test bed of Hamiltonian systems is the standard map, an area-preserving map introduced by Chirikov and Taylor. The standard map is given by

$$
\begin{gathered}
x_{n+1}=x_{n}+\frac{k}{2 \pi} \sin \left(2 \pi y_{n}\right), \\
y_{n+1}=y_{n}+x_{n+1},
\end{gathered}
$$

where $k$ is the parameter controlling integrability. In order to embed the standard map, we only need to replace $T$ and $K(x)$ in Eq. (5) with the appropriate expressions. $T$ stems directly from Eq. (6) and, in accordance with the previous definitions, $K(x)$ becomes

$$
K(x)=\left(\begin{array}{cc}
1 & k \cos \left(2 \pi y_{n}\right) \\
1 & k \cos \left(2 \pi y_{n}\right)+1
\end{array}\right) .
$$

Notice that due to the area-preserving nature of the standard map, the two eigenvalues of the derivative matrix must multiply to 1 . If they are complex, this means that both have an absolute value of 1 , while if they are real, generically one of them will be larger than 1 and the other smaller. We can then separate the phase space into elliptic and hyperbolic regions corresponding to each of these two cases. If a trajectory of the original map lies entirely on the elliptic regions, the overall factor $\exp (-\lambda)$ will damp any small perturbation away from it in the embedded system. But for chaotic trajectories, which inevitably must visit some hyperbolic regions, there exists a value of $\lambda$ such that perturbations away from a standard-map trajectory are amplified instead of dying out in the embedding. As a consequence, such trajectories are ef- fectively expelled from the chaotic regions to settle finally in the safely elliptic KAM islands. This process can be seen clearly in Fig. 1. As the value of $\lambda$ is decreased, the number of trajectories starting from random initial conditions that eventually settle into the KAM tori increases; see Fig. 2.

This process is sensitive enough to work even at hitherto intractably high nonlinearities. For $k=7$, the standard map is almost ergodic: it covers nearly the entire torus with a single chaotic orbit. Only a tiny island of irreductible order resists this invasion. It is located around $(0,0.6774)$ and covers an area approximately a $2 \times 10^{-5}$ part of the torus. Thus, from random initial conditions, one would expect to see it only once every 50000 attempts. Figure 3 shows how easily the bailout method finds this island from simple forward iterates.

A Hamiltonian system does not usually just satisfy volume conservation, but also will conserve the Hamiltonian itself. Given a flow $\dot{x}=f(x)$ with a conserved quantity $E$ $\equiv 0$, then $f \cdot \partial E=0$. However, building a bailout embedding by the procedure above does not lead to dynamics that satisfy $\dot{E} \equiv 0$, because the bailout embedding should be $2 n-2$ dimensional. This is clearly undesirable in the case of Hamiltonian systems, so we show now how to derive a bailout embedding that will obey a conservation law. The bailout equation can be written

$$
\ddot{x}=(\partial f-\lambda) \cdot(\dot{x}-f)+\partial f \cdot \dot{x} .
$$

We need to correct this acceleration so that it stays on the second tangent space of the $E \equiv 0$ surface. Let us call the raw bailout acceleration $u$. The second derivative $\ddot{x}$ has to satisfy $\ddot{x} \cdot \partial E+\dot{x} \cdot \partial \partial E \cdot \dot{x}=0$, so we can modify $u$ to

$$
\ddot{x}=u-\frac{u \cdot \partial E}{|\partial E|^{2}} \partial E-\frac{\partial E}{|\partial E|^{2}} \dot{x} \cdot \partial \partial E \cdot \dot{x} .
$$

This equation, given that we start on $\dot{x} \cdot \partial \mathrm{E}=0$, will then preserve this property.

We have presented a method for control and targeting of chaos in nonlinear dynamical systems: the bailout embedding. While potentially useful in any dynamical-system setting, this method is especially suited to Hamiltonian systems. Unlike other chaos-control strategies, this method does not 
obliterate the original dynamics of the system, but rather preserves it in a privileged slice of phase space embedded in a higher-dimensional space, and merely shifts around the stability of its orbits. A suitable choice of a bailout function allows this strategy to target a complex set of orbits. We have demonstrated, in particular, the targeting of KAM orbits, a case well known from classical studies to be especially hard.
We should like to thank Leo Kadanoff, Rubén Pasmanter, and Marcelo Viana for useful discussions. J.H.E.C. acknowledges the financial support of the Spanish CSIC, Plan Nacional del Espacio Contract No. ESP98-1347. M.O.M. acknowledges the support of the Meyer Foundation. O.P. acknowledges the Spanish Ministerio de Ciencia y Tecnologia, Proyecto CONOCE, Contract No. BFM2000-1108.
[1] E. Ott, C. Grebogi, and J.A. Yorke, Phys. Rev. Lett. 64, 1196 (1990).

[2] Y.-C. Lai, M. Ding, and C. Grebogi, Phys. Rev. E 47, 86 (1993)

[3] E. Bollt and J.D. Meiss, Phys. Lett. A 204, 373 (1995).

[4] C.G. Schroer and E. Ott, Chaos 7, 512 (1997).

[5] Z. Wu, Z. Zhu, and C. Zhang, Phys. Rev. E 57, 366 (1998).

[6] E.E.N. Macau, Phys. Rev. E 57, 5337 (1998).

[7] A. Oloumi and D. Teychenné, Phys. Rev. E 60, 6279 (1999).

[8] Y.L. Bolotin, V.Y. Gonchar, A.A. Krokhin, A. Tur, and V.V. Yanovsky, Phys. Rev. Lett. 82, 2504 (1999).

[9] Y. Zhang and Y. Yao, Phys. Rev. E 61, 7219 (2000).
[10] Y. Zhang, S. Chen, and Y. Yao, Phys. Rev. E 62, 2135 (2000).

[11] H. Xu, G. Wang, and S. Chen, Phys. Rev. E 64, 016201 (2001).

[12] M.C. Gutzwiller, Chaos in Classical and Quantum Mechanics (Springer, Berlin, 1990).

[13] I.C. Percival, in Nonlinear Dynamics and the Beam-Beam Interaction, edited by Melvin Month and John C. Herrera, AIP Conf. Proc. No. 57 (AIP, New York, 1977), p. 302.

[14] A. Babiano, J.H.E. Cartwright, O. Piro, and A. Provenzale, Phys. Rev. Lett. 84, 5764 (2000).

[15] S.C. Venkataramani, B.R. Hunt, E. Ott, D.J. Gauthier, and J.C. Bienfang, Phys. Rev. Lett. 77, 5361 (1996). 